\title{
Nanogels of acetylated ulvan enhance the solubility of hydrophobic drug curcumin
}

\author{
TRUONG HAI BANG ${ }^{1, *}$, TRAN THI THANH VAN ${ }^{1}$, LE XUAN HUNG ${ }^{2}$, BUI MINH LY ${ }^{1}$, \\ NGUYEN DUY NHUT ${ }^{1}$, THANH THUY THU THUY ${ }^{\mathbf{3}}$ and BUI THE HUY $\mathbf{2}^{\mathbf{2} 4}$ \\ ${ }^{1}$ Nhatrang Institute of Technology Research and Application, Vietnam Academy of Science and Technology, Nha Trang \\ 57000, Vietnam \\ ${ }^{2}$ Institute of Research and Development, Duy Tan University, Da Nang 50000, Vietnam \\ ${ }^{3}$ Institute of Chemistry, Vietnam Academy of Science and Technology, Cau Giay Hanoi 10072, Vietnam \\ ${ }^{4}$ Department of Chemistry, Changwon National, Changwon 51140, Republic of Korea \\ *Author for correspondence (truonghaibangnt@gmail.com)
}

MS received 22 November 2017; accepted 26 March 2018; published online 3 January 2019

\begin{abstract}
Curcumin (Cur) is a polyphenol showing various bioactivities including anti-tumour activity. However, poor solubility of Cur in aqueous solution is a major barrier to its bioavailability and clinical efficacy. In this study, hydrophobically modified ulvan was considered to self-assemble to fabricate nanogels. This is a network of hydrophilic segments with hydrophobic microdomains in the nanopores, which keeps Cur from dispersing into the media and raise Cur solubility in water by 20,000 times. A Cur-loaded acetylated ulvan nanogel was characterized by scanning electron microscopy, transmission electron microscopy and dynamic light scattering. This result confirmed that hydrophobically modified ulvan could be used as a self-organizing assembly nanogel to carry and deliver water-insoluble bioactive compounds.
\end{abstract}

Keywords. Nanogel; ulvan; curcumin; green seaweed.

\section{Introduction}

Nanogels are sub-micron three-dimensional networks of cross-linked polymer chains [1]. With a nanoporous structure, nanogels exhibit features of a gel substance and a nano-sized particle, such as high dispersion in aqueous media, biodegradability, biocompatibility, drug loading capacity and increasing drug delivery across cellular barriers [2]. There have been many interesting studies on applying nanogels in drugs, proteins, enzymes and DNA [3-6]. Nanogels can be fabricated from polymer precursors or via heterogeneous polymerization of monomers $[7,8]$. Polymer precursors are amphiphilic or triblock copolymers which can form nanogels by self-assembly or a polymerization process that have number of reactive sites which can be directly used for chemical cross-linking. Of course, polymers can also be modified with other functional groups which can be subsequently used to form physical or chemical cross-links [1].

Ulvan is a sulphated polysaccharide extracted from green seaweed genus Ulva. It is composed of disaccharides of $\mathrm{A}_{3 \mathrm{~S}}$ or $\mathrm{B}_{3 \mathrm{~S}}$. $\mathrm{A}_{3 \mathrm{~S}}$ presents a $\beta$-D-glucuronosyluronic acid- $(1 \rightarrow 4)$ L-Rhap 3-sulphate dimer ( $\beta$-D-GlcpA- $(1 \rightarrow 4)$-L-Rhap 3sulphate) whereas $B_{3 S}$ presents $\alpha$-L-IdopA- $(1 \rightarrow 4)-\alpha$-L-Rhap 3 -sulphate. These unique structures are similar to natural glycosaminoglycans such as chondroitin sulphate and hyaluronic acid. Therefore, ulvan has drawn particular attention to their applications in many fields [9]. Despite the promising properties, the utilization of ulvan in biomedical fields requires structural modification to increase hydrophobic sites on ulvan and prevent macro-gelation. In recent years, based on modified polysaccharide ulvan, there have been very few publications about nanofibre [10] and nanomembrane [11].

Curcumin (diferuloylmethane) (Cur), a hydrophobic polyphenolic compound, is a low molecular-weight active principle of the perennial herb Curcuma longa (commonly known as turmeric). Cur shows a variety of bioactivities such as antioxidant, anti-inflammatory, anti-carcinogenic, anti-microbial and anti-cancer activities but its insolubility in water restricts its use in disease treatment [12]. To date, many techniques have been applied to increase hydrophilic feature of Cur including emulsion, chemical modification and micelle encapsulation [13-17]. However, most of the developed methods require high-operating costs, skilled personnel, time consuming and large capital investment. Therefore, the development of innovatory approach with simple, rapid, low cost and green is an urgent requirement.

Herein, we focus on preparing modified hydrophobically ulvan to encapsulate Cur. Such polysaccharide ulvan from Ulva lactuca, with structures and bioactivities reported in our recent report [18], has been used intentionally as a carrier system to deliver water-insoluble bioactive compounds.

In this study, biocompatible ulvan was modified with acetic anhydride to form amphiphilic polymers. Nanogels based on acetylated ulvan (AcU), prepared by a dialysis method, are 

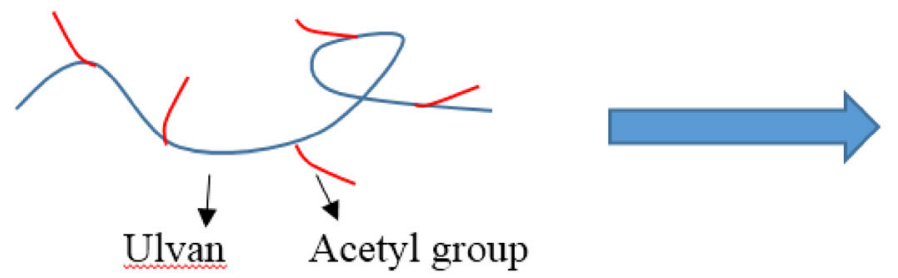

\section{Curcumin}

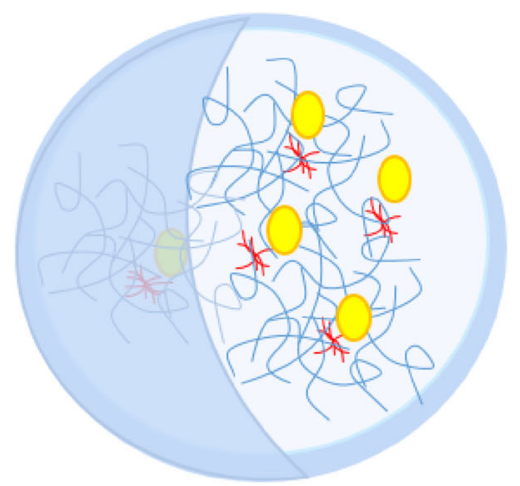

Figure 1. Schematic of self-organized nanogels of ulvan acetate (AcU) in an aqueous medium.

potential to load and deliver hydrophobic compounds namely Cur. A schematic of the concept of self-organizing behaviour of $\mathrm{AcU}$ is shown in figure 1.

\section{Materials and methods}

\subsection{Materials}

Pyridine and acetic anhydride were purchased from Merck (USA) and used without further purification. Pyrene, Cur, acetone and ethyl acetate were purchased from Sigma-Aldrich (USA).

$U$. lactuca samples were collected at the Nha Trang bay in the central coast of Vietnam. The samples were rinsed with seawater followed by pure water and subsequently dried in vacuo. Ulvan was extracted according to the hot-water extraction method by using sodium chlorite [19]. The extract was treated with glucoamylase and protease to remove contaminants. Further purification was accomplished by DEAEcellulose column chromatography followed by dialysis and freeze-drying.

Infrared spectra were recorded using a Fourier-transform infrared spectrophotometer (Jasco FT/IR-6300, Japan). The morphology of the samples was observed using a field emission scanning electron microscope (FE-SEM, MIRAII Tescan, Czech) and a transmission electron microscope (TEM) (JEM-2100F-Jeol, Japan). Cytotoxicity of nanogel AcU-Cur against liver cancer cell lines was also investigated. NMR spectra were recorded on a Bruker Avance 500 in $\mathrm{D}_{2} \mathrm{O}$ solution using DSS as an internal standard at $70^{\circ} \mathrm{C}$.

\subsection{Acetylation of ulvan}

AcU was obtained using a previously reported method [20]. Briefly, $1 \mathrm{~g}$ of ulvan was dissolved in $10 \mathrm{ml}$ of formamide, followed by addition of $1 \mathrm{ml}$ of pyridine and $3 \mathrm{ml}$ of acetic anhydride. After $24 \mathrm{~h}$, the reactant was purified by dialysis for 3 days and then freeze dried. The structure and acetylation degree (AD) of AcU was determined by ${ }^{1} \mathrm{H}-\mathrm{NMR}$. The AD has been calculated by comparing the peak areas relative to the methyl protons of the introduced acetyl groups with the peak areas relative to the methyl groups of the rhamnose present in the native ulvan.

\subsection{Measurement of fluorescence (pyrene)}

Pyrene dissolved in acetone was diluted in distilled water to obtain a concentration of $12 \times 10^{-7} \mathrm{M}$. Acetone was removed under vacuum at $60^{\circ} \mathrm{C}$ for $1 \mathrm{~h}$. The acetone-free pyrene solution and each nanogel solution of different concentrations was mixed and incubated for $6 \mathrm{~h}$ without exposure to light. The final pyrene in each sample solution was $6 \times 10^{-7} \mathrm{M}$. The excitation spectra of the pyrene were recorded at a fixed emission wavelength of $390 \mathrm{~nm}$, and the excitation wavelength was set at $339 \mathrm{~nm}$ for the emission spectra of pyrene which were recorded on a spectrofluorometer (Fluorolog-3-22, JY Horiba Inc., Japan). The slit width was $10 \mathrm{~nm}$ for both excitation and emission.

\subsection{Preparation of Cur-loaded AcU nanogels}

Cur $(5 \mathrm{mg}$ ) was dissolved in $10 \mathrm{ml}$ of anhydrous dimethyl sulphoxide (DMSO) containing $6.2 \mu$ l of triethylamine (TEA) for $12 \mathrm{~h}$ at room temperature. After adding $100 \mathrm{mg}$ of AcU into Cur solution, the mixture was stirred overnight and then dialysed against deionised water for $12 \mathrm{~h}$. The dialysates were kept at $-20^{\circ} \mathrm{C}$ for further experiments.

\subsection{Quantification of Cur extracted from AcU nanogels}

Equal volume of ethyl acetate was added to the Cur AcU nanogel solution and subsequently vortexed for $10 \mathrm{~min}$ and then stirred on a magnetic stirrer overnight. After complete phase separation, the ethyl acetate phase was diluted 10 times, 
and the UV-Vis absorbance at $419 \mathrm{~nm}$ was measured with a UV-Vis spectrophotometer (UV-2505, Labomed, Inc., USA). The quantity of Cur was determined according to the calibration curve of Cur in ethyl acetate in the concentration range of $1-5 \mu \mathrm{g} \mathrm{ml}^{-1}$.

\subsection{Morphological characterization of Cur-loaded AcU nanoparticles}

The hydrodynamic size of the Cur-loaded nanogel was measured using a dynamic light scattering (DLS) system (Mastersizer-2000, Malvern, UK). The autocorrelation intensity was measured at a scattering angle $(\theta)$ of $90^{\circ}$ at $25^{\circ} \mathrm{C}$. All samples were measured in triplicate, and the results were averaged. The morphology of the samples was observed via FE-SEM using a JEM2100F (Jeol, Japan). To prepare the SEM samples, a $10 \mu \mathrm{l}$ aliquot of nanogel solution was dropped on a cover glass and dried for $12 \mathrm{~h}$. Then, it was coated with platinum (15 mA, $45 \mathrm{~s})$.

\section{Results}

\subsection{Ulvan}

Ulvan was extracted according to the hot-water extraction method by using sodium chlorite. Further purification was accomplished by DEAE-cellulose column chromatography. The product of the ulvan extraction process was confirmed by FT-IR spectra, as shown in figure 2. The characteristic absorptions of the sulphate group and uronic acid were identified using the FT-IR spectrum [18]. The absorption peaks at $852,1259,1604$ and $3500 \mathrm{~cm}^{-1}$ were attributed to the $\mathrm{C}-\mathrm{O}-\mathrm{S}$ bending vibration of the sulphate group in the axial position, the $\mathrm{S}=\mathrm{O}$ stretching vibration of the sulphate group, $\mathrm{C}=\mathrm{O}$ of uronic acid and $\mathrm{OH}$ group, respectively.

\subsection{Synthesis of $A c U$}

To increase hydrophobic sites on nanogels, ulvan was chemically modified with acetic anhydride (figure 3 ). AcU samples

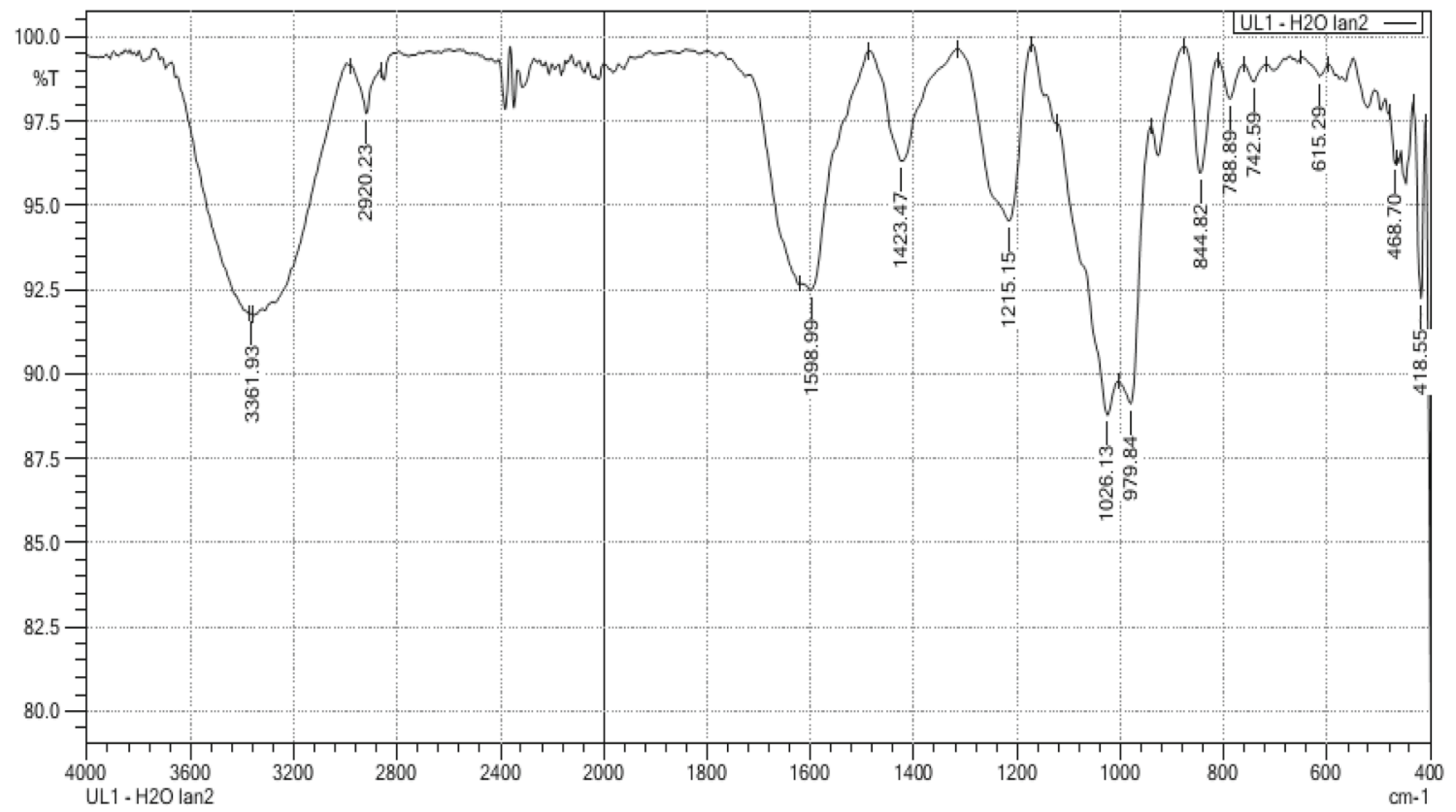

Figure 2. FT-IR spectra of polysaccharide extracted from algae $U$. lactuca.

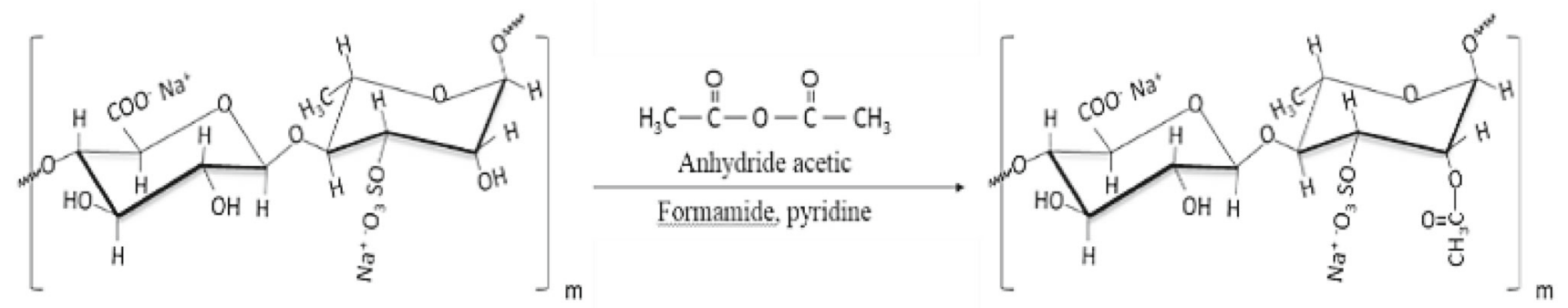

Figure 3. Reaction scheme for the synthesis of AcU. 
were prepared and analysed by ${ }^{1} \mathrm{H}-\mathrm{NMR}$. Figure 4 shows the representative ${ }^{1} \mathrm{H}$-NMR spectrum of the AcU and U. In both spectra, there exist $-\mathrm{CH}$ or $-\mathrm{CH}_{2}$ proton signals of ulvan disaccharide units in the $3-5$ ppm range and the peak at $1.2-$ $1.3 \mathrm{ppm}$ belongs to the methyl group of the rhamnose residue. Typically, only in the AcU spectrum, the intensity of the peak at $1.9 \mathrm{ppm}$ corresponds to the methyl group of the introduced acetyl groups and was much stronger compared with that in the U spectrum. Thus, the data demonstrate that the hydroxyl groups of ulvan were substituted well to the acetyl groups and the $\mathrm{AD}$ was 0.47 .

\subsection{Measurement of critical aggregation concentration (CAC)}

The formation of amphiphilic nanogel particles (hydrophobic-hydrophilic) in polar solvent and the CAC was determined by using a fluorescence spectrophotometer using

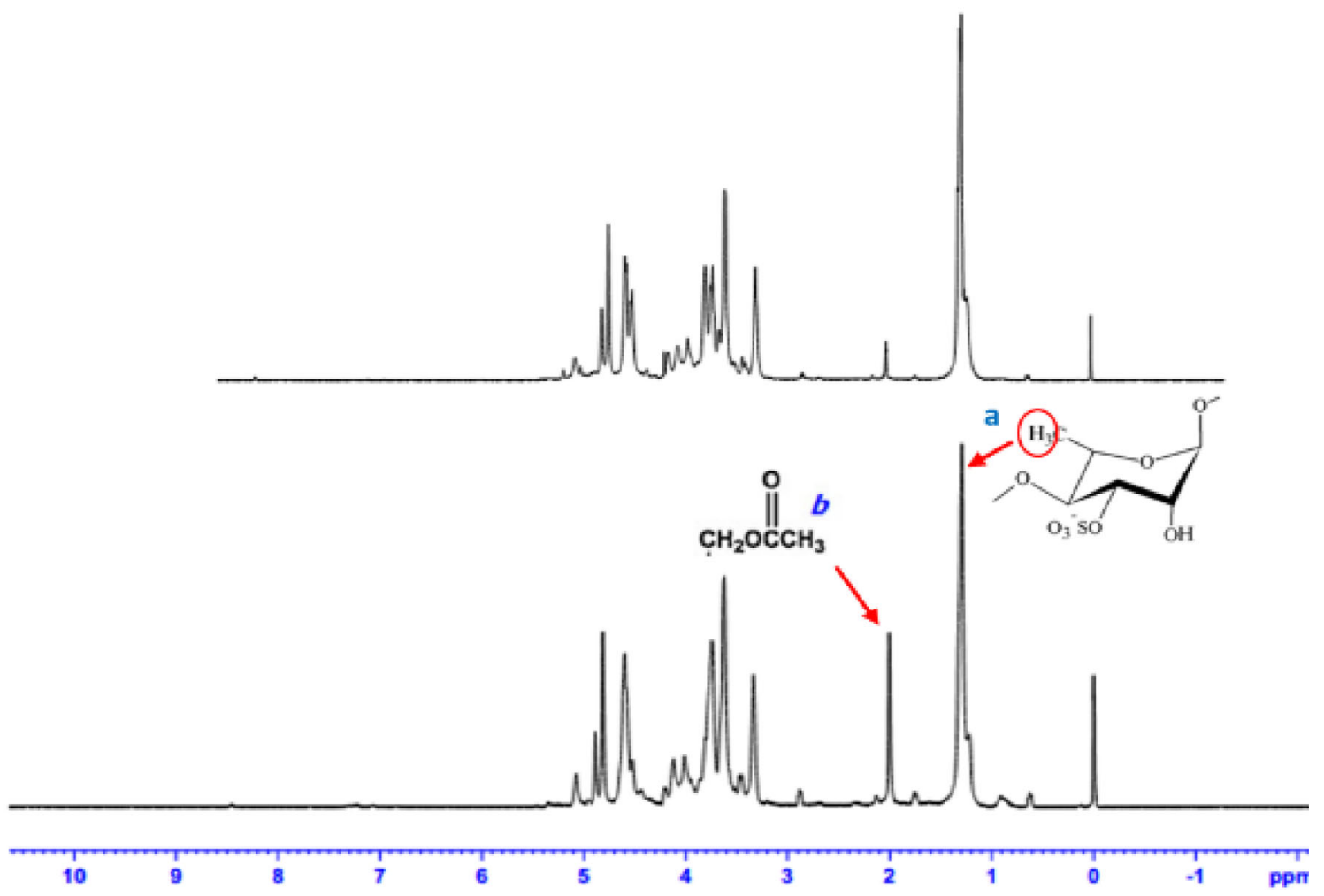

Figure 4. ${ }^{1} \mathrm{H}-\mathrm{NMR}$ spectra of ulvan and AcU.
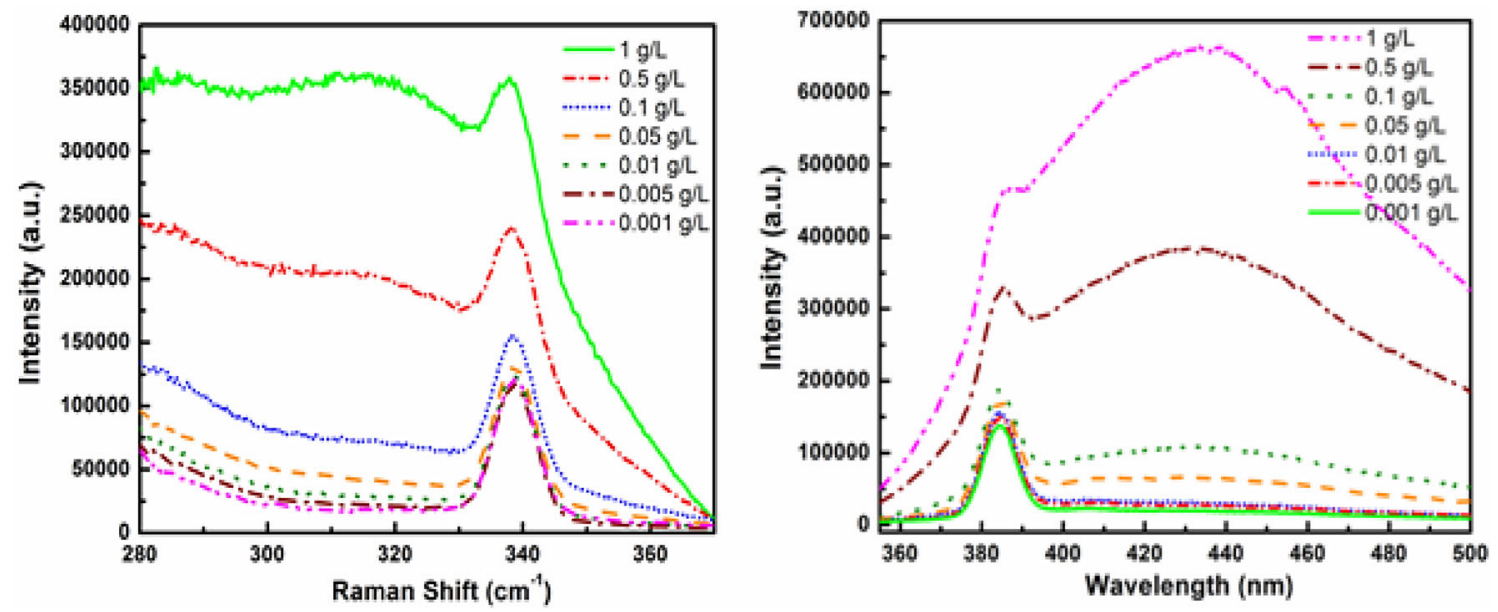

Figure 5. The fluorescence (a) excitation and (b) emission spectra of pyrene at different AcU concentrations. 


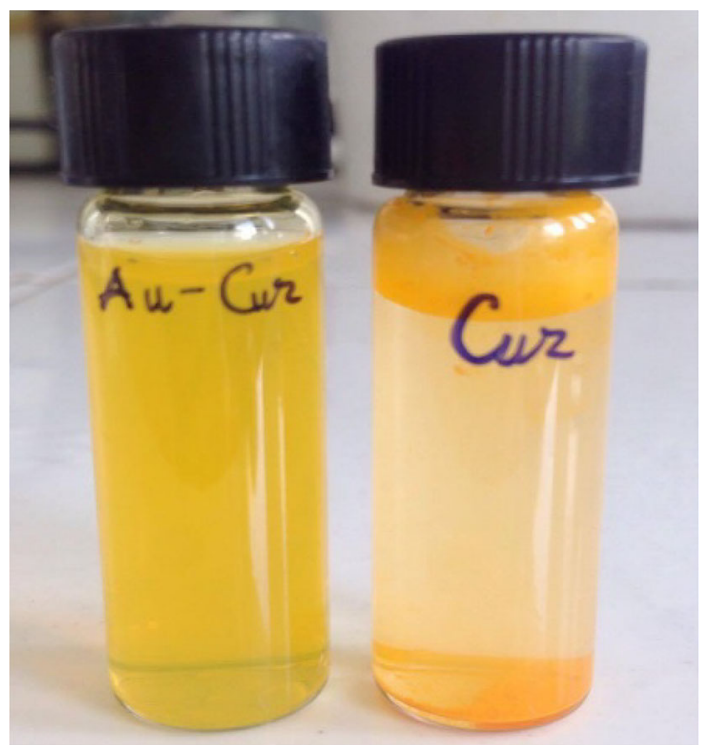

Figure 6. Photographic image of Cur in AcU solution (Au-Cur) and Cur in water. pyrene as a fluorescence probe. According to Lee's report [21], at the concentration that polymer chains interact and form nanogels, pyrene transfers from the polar environment of the solvent to their preferred non-polar region expressed by the shift of the excitation peak from 335 to $339 \mathrm{~nm}$. However, in the excitation spectra (figure 5a) of different $\mathrm{AcU}$ concentrations (even lower than $1 \mathrm{mg}^{-1}$ ), there merely exist the excitation peak at the wavelength of $339 \mathrm{~nm}$. This indicates that at low AcU concentrations $\left(\mathrm{CAC}<1 \mathrm{mg} \mathrm{l}^{-1}\right.$ ), nanogel was created. The $\mathrm{CAC}$ value of $\mathrm{AcU}$ is lower than those of other natural polymers such as chondroitin sulphate acetate and fucoidan acetate $\left(1.9 \times 10^{-2} \mathrm{mg} \mathrm{ml}^{-1}\right.$ and $0.039 \pm 0.002 \mathrm{mg} \mathrm{ml}^{-1}$, respectively) [20-23]. This could be explained by the difference in the $\mathrm{AD}$ of different polymers.

At a fixed excitation wavelength of $339 \mathrm{~nm}$, fluorescence emission spectra of pyrene at different AcU concentrations were recorded (figure 5b). The high intensity of a symmetric peak at $390 \mathrm{~nm}$ exhibits strong emission of pyrene which is captured by hydrophobic microdomains on nanogels. In particular, there is a broad band (bandwidth spreads from 390 to $490 \mathrm{~nm})$ at high concentrations of $\mathrm{AcU}\left(>100 \mathrm{mg} \mathrm{l}^{-1}\right)$, a
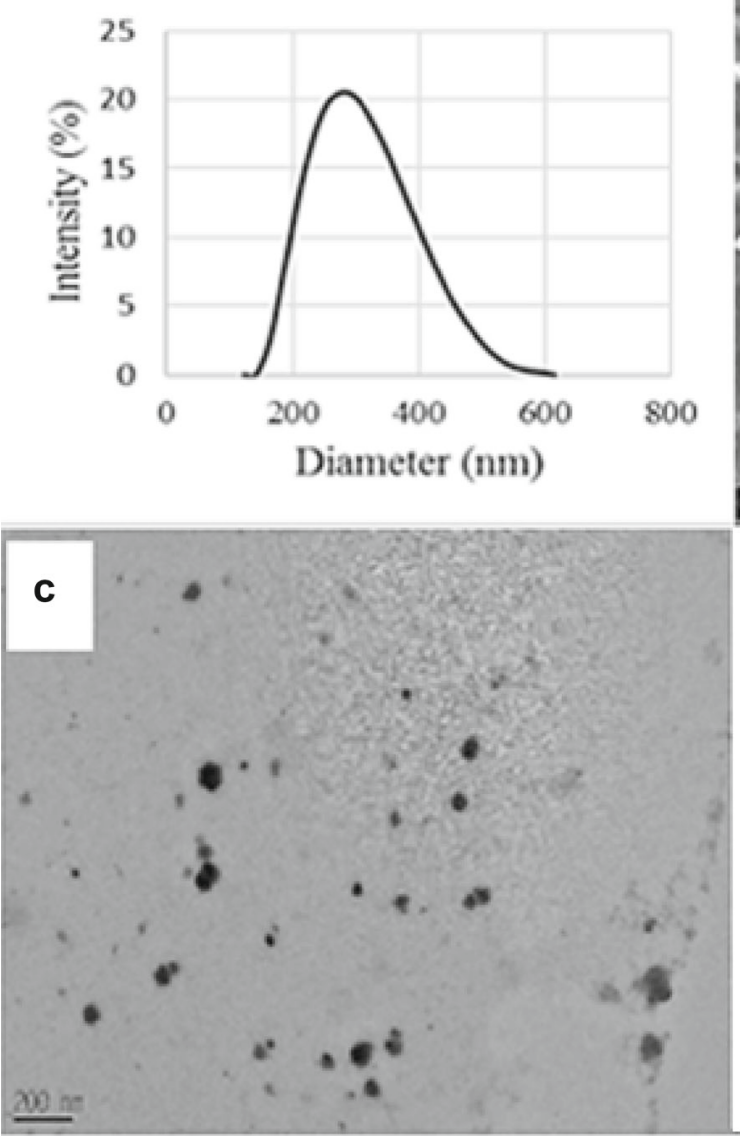
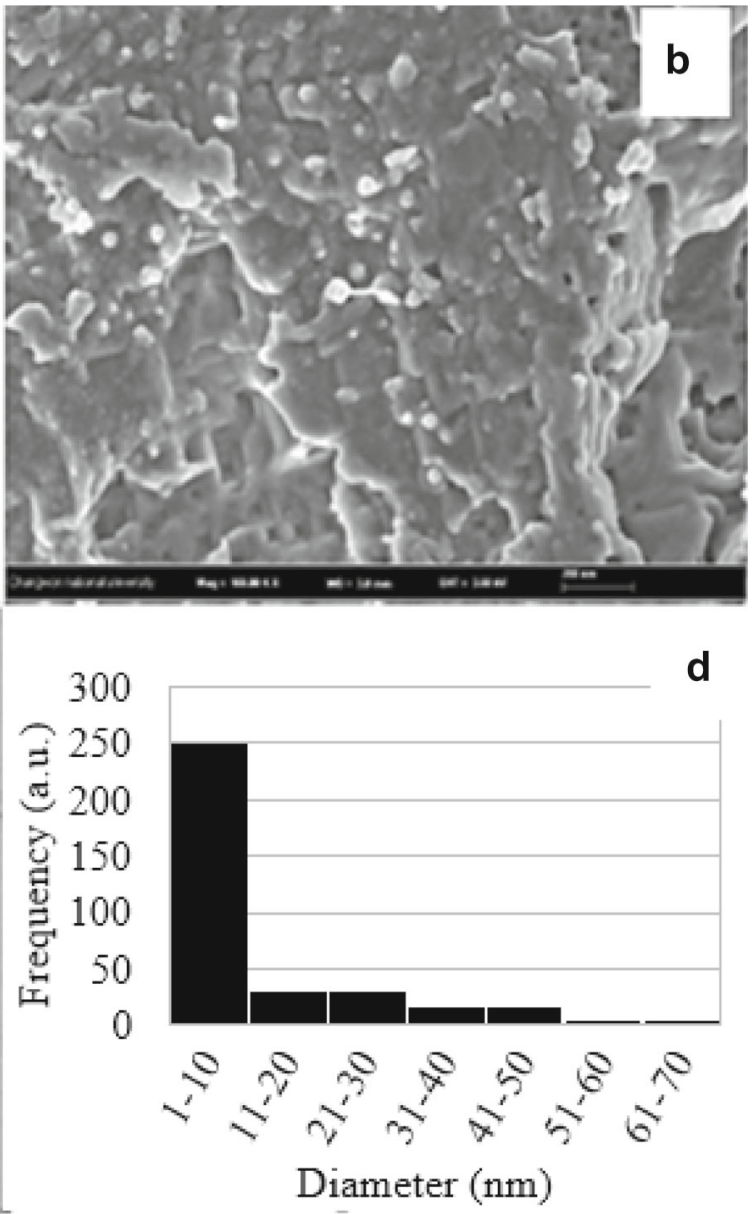

Figure 7. (a) Diameters, (b) FE-SEM image, (c) TEM image of AcU nanoparticles and (d) diameter distribution of Cur on AcU. 


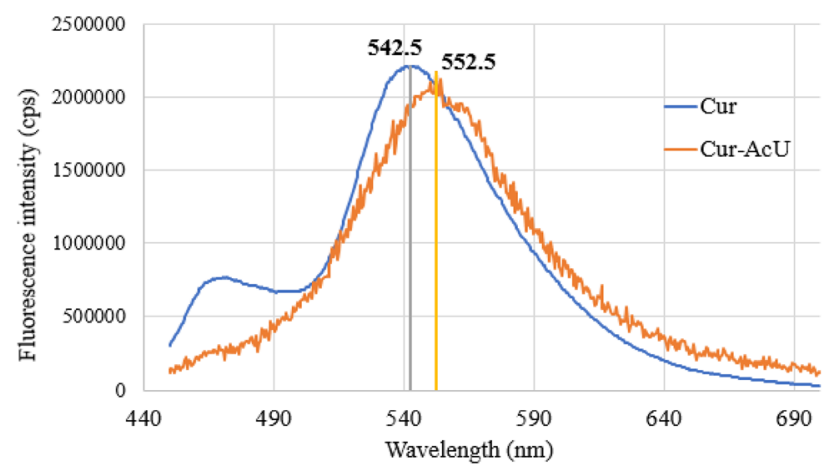

Figure 8. Fluorescence emission spectra of Cur and Cur-AcU in water.

which expresses the tendency of gel membrane formation in solution [24]. Therefore, to avoid formation of unexpected gel membrane, AcU concentration must be prepared lower than $100 \mathrm{mg} \mathrm{l}^{-1}$ to maintain nanogel particles in solution.

\subsection{Preparation and characterization of Cur-loaded AcU nanogels}

Cur was loaded on AcU nanogels by the dialysis method. During dialysis, the solution underwent a change in turbidity from clean to opaque because of the formation of nanogels. After lyophilization, the obtained yellow powder was soluble easily in water with no noticeable Cur precipitates, as shown in figure 6 .
Our results suggested that Cur was indeed trapped by $\mathrm{AcU}$ nanogels and the complex of AcU and Cur could resist against the freeze-drying process.

Cur in nanogel AcU was quantified from an ethyl acetate extract; the concentration of Cur was calculated by using the calibration curve of Cur in ethyl acetate. Thus, the concentration of Cur in a solution of $1 \% \mathrm{AcU}$ was $236 \mu \mathrm{g} \mathrm{ml}^{-1}$, which was nearly 20,000 times higher than its concentration in water (11 $\mathrm{ng} \mathrm{ml}^{-1}$ ) [25] and nearly 13 times higher than its concentration in $1 \%$ hydrophobically modified starch $\left(18.4 \mu \mathrm{g} \mathrm{ml}^{-1}\right)$ [14]. This outstanding solubility could be the results of hydrogen bonding between the Cur and hydrophobic sites of AcU. This type of interaction between the Cur and other substances, such as phosphatidylcholine, has also been mentioned in the previous report [26].

From DLS measurements (figure 7a), only one sharp peak on the spectrum showed the average swollen diameter of the Cur-loaded nanogel as $293 \mathrm{~nm}$. The $P_{\mathrm{di}}$ value of the diameter was $0.220 \pm 0.013 \mathrm{~nm}$. The value of the zeta potential was $-28.6 \mathrm{mV}$, reflecting the anion charge repulsion among ionic carboxyl and sulphonic acid of nanogel particles.

To visualize the morphology of the nanogel particles, FESEM and TEM techniques were applied. It is unable to observe the dispersion of nanogel particles in solution since samples were obliged to be desiccated for the preparation process, the FE-SEM image (figure 7b) still exhibits the surface morphology of the gel with many nanopores and circular spots. Instead of relatively ineffective towards organic materials, in fact, TEM results of diluted solution (figure 7c) and statistic report of the dark spot diameters from SEM image (figure $7 \mathrm{~d}$ ), the circular spots (with diameter mostly lower than $10 \mathrm{~nm}$ ) were referred to the Cur used.

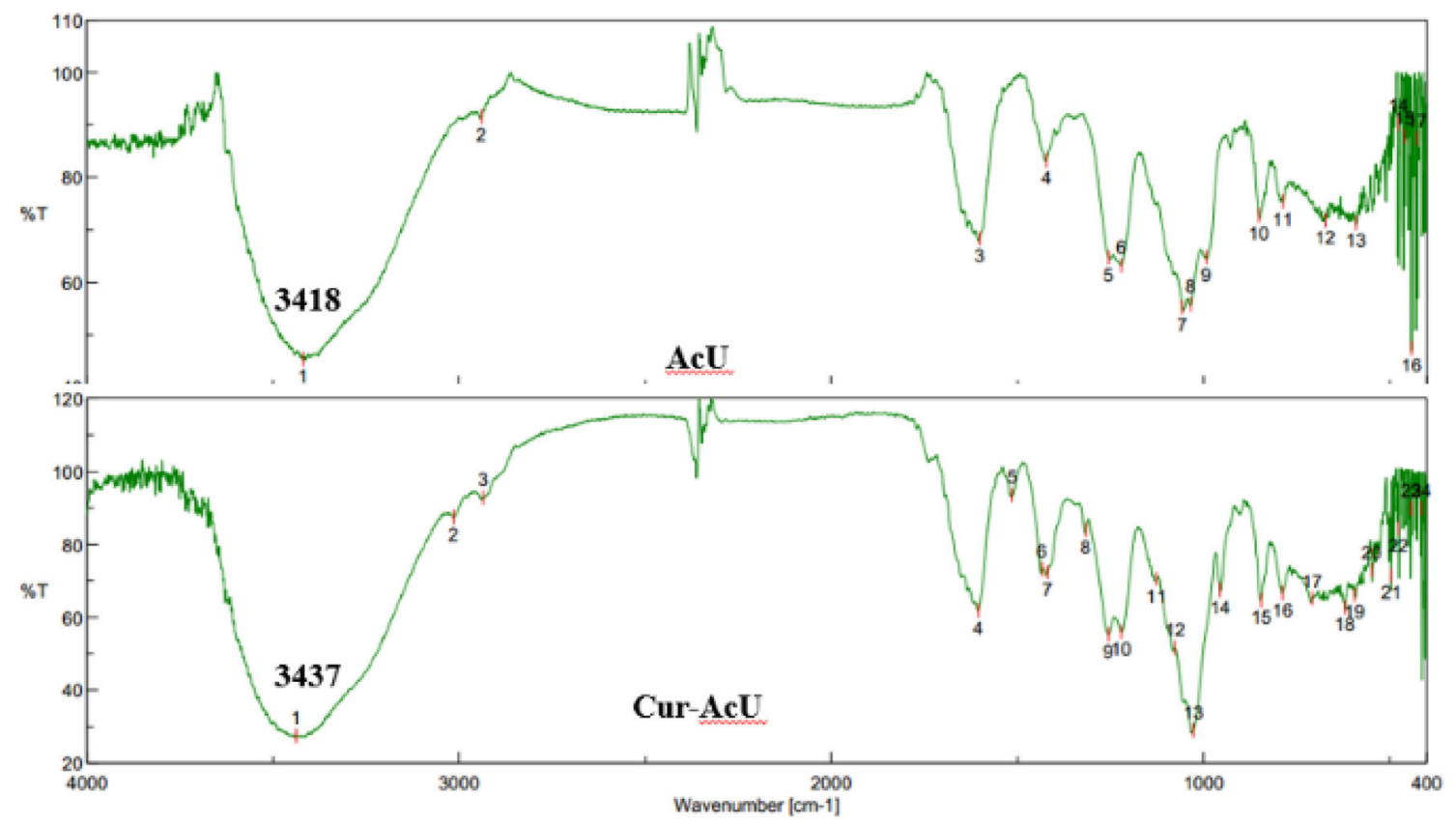

Figure 9. IR spectra of AcU and Cur-AcU. 
To further determine linking formation of encapsulated Cur and nanoulvan particles, fluorescence emission spectra of Cur and Cur-AcU in water were recorded after excitation at $420 \mathrm{~nm}$. As shown in figure 8, the emission peak of Cur is at $542.5 \mathrm{~nm}$. It is shifted to $552.5 \mathrm{~nm}$ upon the encapsulation by AcU. This seems to come from the formation of intermolecular hydrogen bonding between the Cur and AcU, which can be explained by IR data of AcU nanoparticles and Cur-AcU. Compared with that of pure AcU, the IR spectrum of Cur-AcU shows a band shift from 3418 to $3437 \mathrm{~cm}^{-1}$ (figure 9), which corresponds to hydrogen bonding between the $-\mathrm{OH}$ groups of Cur and $\mathrm{AcU}$.

In summary, these results confirmed the homologous dimension of the nanogel particles in the form of a network of hydrophilic segments with hydrophobic microdomains in the nanopores, which keep Cur from dispersing into the media.

Thereby, these AcU nanogels acted as an effective template contributing to effective dispersion of nanosized Cur and prevented Cur nanoparticles from re-aggregating. By this, Cur can be dissolved considerably in water. The nanogels can be used as delivery systems for hydrophobic substances including advanced drugs and proteins.

\section{Conclusion}

Acetylated polysaccharide ulvan is able to self-assemble to form nanogels with $\mathrm{CAC}<1 \mathrm{mg} \mathrm{l}^{-1}$. Being loaded onto nanogel AcU, Cur showed the solubility increased by 20,000 times. This result confirmed that hydrophobically modified ulvan could be used as a self-organizing assembly nanogel to carry and deliver water-insoluble bioactive compounds.

\section{Acknowledgement}

This research is funded by the Vietnam Academy of Science and Technology (Project number VAST03.04/15-16).

\section{References}

[1] Yallapu M M, Jaggi M and Chauhan S C 2011 Drug Discovery Today $\mathbf{1 6} 457$
[2] Soni G and Yadav K S 2016 Saudi Pharm. J. 24133

[3] Toita S, Sawada S-I and Akiyoshi K 2011 J. Control Release 15554

[4] Aimetti A A, Machen A J and Anseth K S 2009 Biomaterials 30 6048

[5] Catarina G, Paula P and Miguel G 2010 Materials 31420

[6] Le P N, Nguyen N H, Nguyen C K and Tran N Q 2016 Bull. Mater. Sci. 391493

[7] Zhang H, Zhai Y, Wang J and Zhai G 2016 Mater. Sci. Eng. $C 60560$

[8] Vaghani S S, Patel M M, Satish C S, Patel K M and Jivani N P 2012 Bull. Mater. Sci. 351133

[9] Federica C and Morelli A 2011 In: R Pignatello (ed) Biomaterials (Rijeka: IntechOpen) chapter 4

[10] Toskas G, Hund R-D, Laourine E, Cherif C, Smyrniotopoulos V and Roussis V 2011 Carbohydr. Polym. 841093

[11] Toskas G, Heinemann S, Heinemann C, Cherif C, Hund R-D, Roussis V et al 2012 Carbohydr. Polym. 89997

[12] Das R K, Kasoju N and Bora U 2010 Nanomed. Nanotechnol. 6 153

[13] Sari T P, Mann B, Kumar R, Singh R R B, Sharma R, Bhardwaj $\mathrm{M}$ et al 2015 Food Hydrocoll. 43540

[14] Mohamed S A, El-Shishtawy R M, Al-Bar O A M and AlNajada A R 2017 Int. J. Food Prop. 20718

[15] Alizadeh A M, Sadeghizadeh M, Najafi F, Ardestani S K, Erfani-Moghadam V, Khaniki M et al 2015 BioMed. Res. Int. 201514

[16] Yu H and Huang Q 2010 Food Chem. 119669

[17] Rajan S S, Pandian A and Palaniappan T 2016 Bull. Mater. Sci. 39811

[18] Thanh T T T, Quach T M T, Nguyen T N, Vu Luong D, Bui M L and Tran T T V 2016 Int. J. Biol. Macromol. 93695

[19] Robic A, Sassi J F and Lahaye M 2008 Carbohydr. Polym. 74 344

[20] Park W, Park S-J and Na K 2010 Colloids Surf. B 79501

[21] Lee C-T, Huang C-P and Lee Y-D 2006 Biomacromolecules 7 1179

[22] Likhitwitayawuid K, Angerhofer C K, Cordell G A, Pezzuto J M and Ruangrungsi N 1993 J. Nat. Prod. 5630

[23] Lee K W, Jeong D and Na K 2013 Carbohydr. Polym. 94850

[24] Mazor S, Vanounou S and Fishov I 2012 Chem. Phys. Lipids 165125

[25] Tønnesen H H, Másson M and Loftsson T 2002 Int. J. Pharm. 244127

[26] Xie J, Li Y, Song L, Pan Z, Ye S and Hou Z 2017 Drug Delivery 24707 